\title{
Decoding the Molecular Evolution of Human Cognition Using Comparative Genomics
}

\author{
Noriyoshi Usui Marissa Co Genevieve Konopka \\ Department of Neuroscience, University of Texas Southwestern Medical Center, Dallas, Tex., USA
}

\section{Key Words}

Human brain evolution - FOXP2 - Autism - Autism spectrum disorder - Cognitive diseases - Cognition - Comparative genomics - Weighted gene coexpression network analysis

\begin{abstract}
Identification of genetic and molecular factors responsible for the specialized cognitive abilities of humans is expected to provide important insights into the mechanisms responsible for disorders of cognition such as autism, schizophrenia and Alzheimer's disease. Here, we discuss the use of comparative genomics for identifying salient genes and gene networks that may underlie cognition. We focus on the comparison of human and non-human primate brain gene expression and the utility of building gene coexpression networks for prioritizing hundreds of genes that differ in expression among the species queried. We also discuss the importance of and methods for functional studies of the individual genes identified. Together, this integration of comparative genomics with cellular and animal models should provide improved systems for developing effective therapeutics for disorders of cognition.
\end{abstract}

C 2014 S. Karger AG, Basel

0006-8977/14/0842-0103\$39.50/0

\section{Introduction}

The evolution of the human brain has permitted great advantages in cognitive capacities. However, converging evidence suggests that a deleterious consequence of these advances has been the emergence of cognitive disorders, both neurodevelopmental (e.g. autism and schizophrenia) and neurodegenerative (Alzheimer's disease) [Crow, 2000, 2008; Teffer and Semendeferi, 2012; Bruner and Jacobs, 2013; Raichlen and Alexander, 2014]. Thus, by better understanding human brain evolution, more progress will be made into uncovering the etiologies of these cognitive disorders.

Genetic association has arisen as one of the most productive strategies for deciphering the pathophysiology of cognitive disorders due to the genomic revolution, generating faster and cheaper technologies for DNA sequencing and the significant contribution of genetics to most of these disorders. Unfortunately, the genetics of cognitive disorders do not typically follow straightforward Mendelian patterns, but rather point to a complex, multigenic landscape potentially interacting with epigenetic and/or environmental factors in most cases [Berg and Geschwind, 2012; Kim and State, 2014; Krumm et al.,

\section{KARGER}

E-Mail karger@karger.com www.karger.com/bbe
Genevieve Konopka, $\mathrm{PhD}$

Department of Neuroscience, University of Texas Southwestern Medical Center 5323 Harry Hines Boulevard, ND4.300 Dallas, TX 75390-9111 (USA)

E-Mail genevieve.konopka@ utsouthwestern.edu 
2014]. Such genetic profiles make modeling these diseases for therapeutic discovery even more challenging. Thus, the use of bioinformatic approaches for prioritizing genes for further study should prove useful in this regard.

Typical workflows for elucidating therapeutic pharmacological compounds include assaying function, efficacy and safety using animal models. A caveat to the study of cognitive disorders in model systems is the idea that many of these disorders are unique to humans [Crespi et al., 2007; Lepp et al., 2013]. Some of this argument is tautological due to the inclusion of disruption to human-specific traits such as 'language' as part of the diagnosis. Therefore, it would be virtually impossible to define such a disorder in another species, such as our closest living relatives the chimpanzees. Also, given the limitations we have in observing large numbers of nonhuman primates in either natural or laboratory settings, we will likely always be underpowered for observing similar phenotypes in these species. Therefore, the majority of investigators in the field must make the assumption that the constellations of phenotypes, both behavioral and pathological, that occur in each of these disorders are unique to humans. However, the human brain was not built from scratch, and much of the brain's basic circuitry and molecular mechanisms are conserved at least back to commonly used laboratory models such as rodents. Much can therefore be learned about these conserved neurobiological processes by studying the brains of mice or rats [Oh et al., 2014]. Furthermore, much can be learned about specific genetic molecular mechanisms in these model systems. Unfortunately, however, drugs that 'cure' cognitive disease-like behavior or pathology in rodent models often fail to ameliorate symptoms in patients during clinical trials (e.g. solanezumab or bapineuzumab for the treatment of Alzheimer's disease) [Doody et al., 2014; Salloway et al., 2014]. The use of information about what makes a human brain unique in the design of these animal models could potentially increase the efficacy of future drug studies.

The methods to obtain and define potential human brain-unique features are diverse. Here, we assert that by studying changes in gene expression unique to the human brain we can identify salient genomic features relevant to both brain evolution and cognitive diseases. This logic follows from the observation of disruption to human cognitive specializations in such diseases and the significant overlap of genes identified in cognitive diseases with those that show evolutionary changes in expression, specifically in the human brain. Two major approaches are discussed. The first approach is to focus on single genes with known association to human specializations, such as language or cognitive disorders. Thus, we will detail findings pertinent to the gene encoding FOXP2, which has been linked to language, autism and schizophrenia, and has also undergone important changes in the human lineage from a molecular evolution perspective [Lepp et al., 2013]. The second approach is to take a broad hypothesis-generating strategy through the use of comparative genomics from brain tissue of humans and non-human primates. Using advanced genomic techniques, such as RNA sequencing (RNA-seq), combined with bioinformatic prioritization techniques, such as weighted gene coexpression network analysis (WGCNA), can lead to the identification of novel human brain expression patterns that may be important for cognition [Konopka and Geschwind, 2010]. Using both of these approaches can be most effective for identifying patterns of gene expression unique to the human brain. What remains the most challenging step is how to study the function of these human patterns in pertinent model systems in order to provide insight into both cognition and disorders of cognition. We therefore present the use of both human cellular models as well as humanized animal models as the most suitable options for functional follow-up studies.

\section{Decoding Cognitive Disorders Using an Evolutionary Perspective}

\section{Human Brain Evolution and Autism}

Cognitive disorders such as autism or schizophrenia are often thought of as human-specific disorders due to the disruption of higher cognitive functions of the brain. Autism, or autism spectrum disorder (ASD), is a neurodevelopmental disorder with complex genetics, characterized by impaired communication and social interactions, as well as repetitive or stereotyped behaviors and interests [American Psychiatric Association, 2000; Toro et al., 2010; Bowers and Konopka, 2012; American Psychiatric Association, 2013]. The prevalence of ASD overall is approximately 1 in 100 in the USA, and 1 in 160 worldwide, making ASD a major public health problem around the world [Baird et al., 2006; Fernell and Gillberg, 2010; Elsabbagh et al., 2012]. Similar to other neuropsychiatric disorders, one of the features of ASD is the occurrence of the disorder approximately four times more frequently in males than females [Chakrabarti and Fombonne, 2001; Fombonne, 2005; Robertson, 2008; Werling and Geschwind, 2013]. 
There have been many insights made into various biological underpinnings of ASD. Many of the genes that are responsible for this disorder still remain mostly unknown; however, recent sequencing studies have predicted that over 1,000 genes may be involved [Sanders et al., 2012]. Genetics has proven especially powerful to investigate the potentially diverse molecular mechanisms underlying ASD. The genetic architecture of ASD is highly heterogeneous and likely includes the combinations of alleles with high or low penetrance [Berg and Geschwind, 2012; Krumm et al., 2014]. In addition, alterations in one or more of these genes occur in different combinations in each individual with ASD, leading to this diverse genetic architecture and perhaps partly underlying the spectrum of behavioral phenotypes. One biological mechanism underlying ASD etiology for which there is the strongest support, at least at the genetic level, is dysfunction at the synapse and synaptic function [Flavell et al., 2006; Kelleher and Bear, 2008; Toro et al., 2010]. In addition, the broad expression and function of ASD causative genes in the brain suggests that alteration of synaptic homeostasis could be a biological process associated with ASD [Toro et al., 2010]. Moreover, other altered biological processes, such as developmental delay, synapse structure, neuronal connectivity and neural circuits, underlie ASD [Berg and Geschwind, 2012]. By studying these changes, important insights will be made into human brain evolution as well as these cognitive disorders. As language and communication are disrupted in ASD, and language is a human-specific trait, these studies should provide insight into the genetic and molecular pathways underlying language. In addition, the disruption to social cognition and theory of mind (ToM) in ASD (as well as schizophrenia and other cognitive disorders) [King and Lord, 2011] likely has evolutionary underpinnings as ToM is thought to have undergone specialization or sophistication in the human brain, along with other social capacities [Shettleworth, 2012; Seyfarth and Cheney, 2013]. Therefore again, gaining insight into circuits and mechanisms at play in ToM will not only provide information relevant to ASD, but also to human brain evolution.

\section{Current Approaches of ASD Research}

To address the molecular mechanisms underlying ASD and other cognitive disorders, a 'traditional' approach has been to take patient samples (typically blood), identify gene mutations, and then make a similarly modified mouse. These mice demonstrate construct validity if they carry a mutation in a gene that has some support

Decoding the Molecular Evolution of Human Cognition in the human genetics literature. Also, face validity reflects the fact that the mouse bears some physical or behavioral resemblance to the human disorder. Finally, predictive validity indicates a similar response to a therapy that is known to be effective in people [Silverman et al., 2010; Crawley et al., 2012] (fig. 1a). As the diagnostic criteria for autism are behavioral, phenotyping these mouse models requires behavioral assays with high relevance to each category of the diagnostic symptoms. For example, a comprehensive set of assays for social interaction, communication and repetitive behaviors is used. Based on many previous studies, mouse models hold great promise as translational tools for discovering effective treatments for components of ASD [Silverman et al., 2010]. Many of the well-characterized mouse models of ASD have some degree of construct and face validity, and occasionally have predictive validity. However, a major problem for using the mouse for these kinds of basic and translational studies is that there are many differences between human and mouse brains, including morphology and function. For example, aside from the obvious difference in relative brain size between humans and mice, specialized pyramidal neurons (von Economo neurons) in the anterior cingulate cortex and frontoinsular cortex are not found to be morphologically similar in the mouse brain, if indeed they are comparable cells [Allman et al., 2010], neuronal dendritic spine density does not vary among cortical regions in mice, unlike that which is found in primates [Ballesteros-Yanez et al., 2006], and hemispheric lateralization that results in a functional outcome, such as paw preference, is limited in the mouse [Li et al., 2013]. Thus, it is not entirely surprising when clinical trials based on studies in mice fail in people. The mouse as a model system is still useful, but we assert that using an evolutionary approach will give this model increased power.

\section{Evolutionary Approaches to Understanding Human-} Specific Cognitive Disorders

Language is arguably the most compelling and unique feature of human evolution, and as discussed above it is also disrupted in many cognitive disorders [Ullman, 2001]. How can we uncover the origins of human language at a genetic and molecular level and obtain effective insights into neurodevelopmental disorders such as autism from this information? Many previous studies of human brain evolution have focused on neuroanatomical and behavioral comparisons. These approaches are necessary for building the context upon which molecular and cellular comparisons can be placed. We and others are 
Fig. 1. An evolutionary approach to investigating human-specific cognitive disorders. a A typical approach for studying cognitive disorders is to identify the responsible genes and/or mutations using patient data. Genes and/or mutations identified are then analyzed using a mouse model, and elucidation of pathogenesis and treatment is established using the mouse. $\mathbf{b}$ In contrast, an evolutionary approach is based on comparative genomics from humans and other primates. From these studies, human specializations are identified and phenotypes focusing on genomics, development, neuronal differentiation and activity, and behaviors using human cells and mouse models are analyzed. Eventually, these human specializations are generated in humanized animals and genes important in cognitive disorders can be simultaneously manipulated to develop novel treatments.

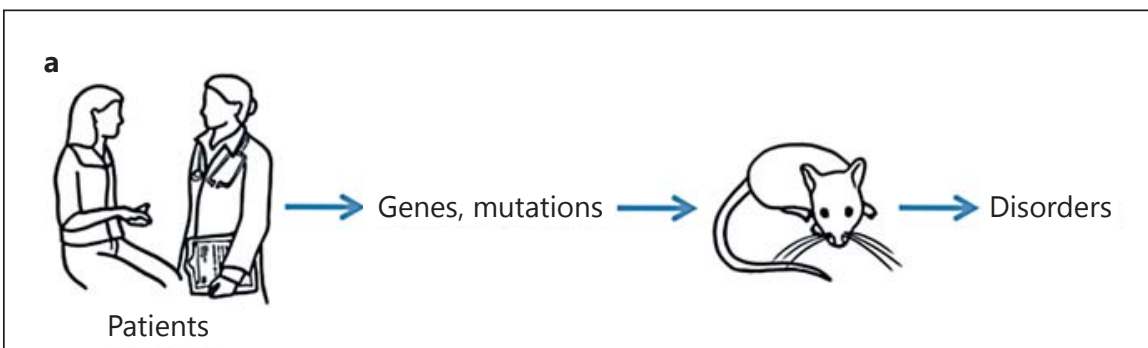

b

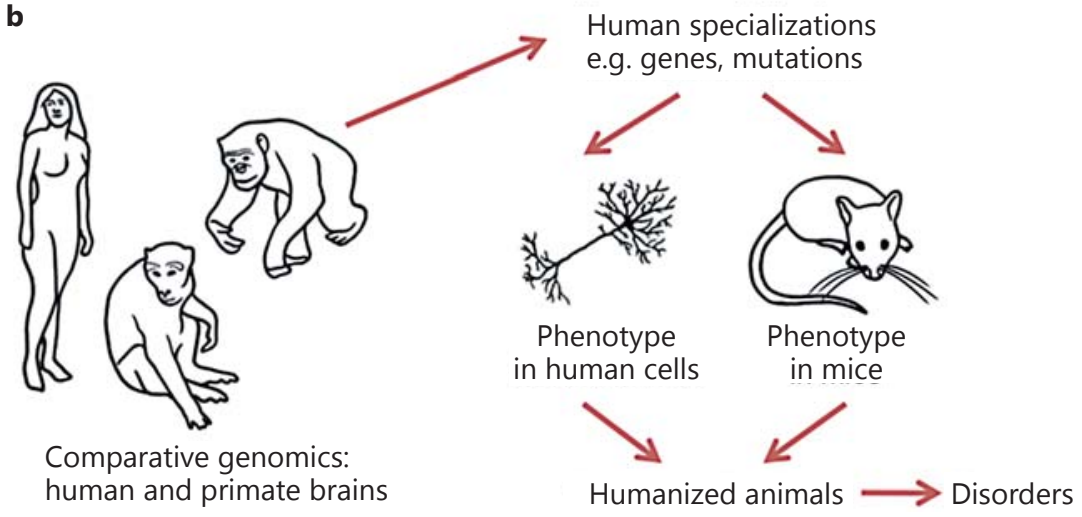

trying to decode the molecular evolution of human brain evolution and cognition by collating comparative gene expression and regulation data with neuroanatomical and behavioral data [Konopka and Geschwind, 2010; Buckner and Krienen, 2013; Somel et al., 2013]. Here, we discuss insights into the genetics and genomics of human brain evolution generated through the use of technological advances in sequencing.

One strategy is a candidate approach. Can changes to a single gene dramatically alter a complex human trait? As discussed below, a prime candidate, FOXP2, is associated with language and speech, and may be involved in brain mechanisms underlying human-specific features and brain evolution [Lai et al., 2001; Fisher et al., 2003]. It is possible to assess the evolution of single genes by comparing nucleotide changes across multiple species [Chimpanzee Sequencing and Analysis Consortium, 2005; Konopka and Geschwind, 2010]. Moreover, to investigate human evolution, focusing on the genes with human-specific modifications should be an effective evolutionary approach (fig. 1b).

The second strategy is a hypothesis-generating approach. This approach to human brain evolution and cognitive disorders works from the hypothesis that vul- nerability to disorders of cognition emerged as a consequence of the evolution of cognition itself. In contrast to other approaches, this strategy uses evolutionary information such as comparative genomics conducted with high throughput sequencing technologies and humanized transgenic animals (fig. 1b). High throughput sequencing technologies known as next-generation sequencing (NGS) include techniques such as chromatin immunoprecipitation coupled to DNA sequencing (ChIP-seq), RNA-seq and de novo genome assembly, and they can be used to view human genome evolution at high resolution and to identify novel coding and noncoding information for regulation of gene expression in the human lineage [Konopka and Geschwind, 2010; Somel et al., 2013; Telese et al., 2013]. Thus, NGS facilitates the comparison of genomes among different species for evolutionary comparisons relevant to human-specific cognitive disorders. Changes in DNA sequences, gene expression, noncoding RNAs and functionally relevant ontological categories can all be discovered as specific to one species. To examine the function of the identified genes relevant to human brain evolution, cultured neuronal cells and human neural progenitors for in vitro assays [Konopka et al., 2012a], and transgenic, humanized ani- 
mals, such as mouse, rat and marmoset, for in vivo analysis, are now available. These humanized animals contain a genetic alteration that mimics the human gene and differs from the endogenous version of the gene. An example is humanized FOXP2 mice which have one of the mouse Foxp2 exons replaced genetically with a human exon, resulting in two amino acid changes in the mouse Foxp2 to mimic human FOXP2 [Enard et al., 2009]. In particular, humanized mice are a powerful and useful tool for understanding human gene function in vivo due to the cost and available tools and datasets relevant to mice (fig. 1b). Such advances will transform our understanding of many neurological processes, including novel findings of neuronal identity, diversity and connectivity in the human brain. However, many of these studies need to be designed and interpreted with caution. For example, other changes dependent on the species' overall genetic profile may be necessary for functioning of the human variant to mimic human patterns. One could imagine cofactor expression and splicing variation in cell type-specific manners across species playing an important determinant on outcome. The generation of animals with multiple humanized genes may alleviate some of these issues, but most likely not all.

\section{FOXP2 in Human Brain Evolution and Cognition}

\section{Involvement of FOXP2 in Speech and Language, a Hallmark of Human Evolution}

The gene encoding forkhead box P2, or FOXP2, was first identified in the KE family, a large multigenerational family with an inherited speech and language phenotype [Lai et al., 2001]. Mutations in the gene encoding FOXP2 have been linked to speech and language through alterations in the ability of patients to complete complex orofacial sequential movements required for normal speech [Lai et al., 2001; Marcus and Fisher, 2003; Vargha-Khadem et al., 2005; Bacon and Rappold, 2012]. With the exception of echolocating bats where FoxP2 has undergone accelerated evolution in regions of the protein with unknown functional significance [Li et al., 2007], FOXP2 is highly conserved among mammals, but two novel amino acids, T303N and N325S, arose in the protein sequence when the common ancestor of humans and chimpanzees diverged [Enard et al., 2002]. The timing of these changes suggests that these two alterations in the human protein may have contributed to an acceleration in the evolution of FOXP2 functions, including the mechanisms underlying acquisition of language and speech [Enard et al., 2009;

Decoding the Molecular Evolution of Human Cognition
Konopka et al., 2009] (fig. 2a). While it would be interesting to hypothesize as to why FoxP2 has undergone accelerated evolution in two lineages (bats and primates) in two distinct areas of the protein, the lack of understanding of the brain circuitry underlying language and echolocation and how to compare these behaviors would make any hypothesis extremely speculative at this point. So, we here discuss FOXP2 function as a unique human feature within the latest view of human brain evolution.

The two 'human-specific' amino acid changes in FOXP2 discussed above were also confirmed in Neanderthals [Krause et al., 2007; Green et al., 2010] and Denisovans [Reich et al., 2010], indicating that the molecular evolution of FOXP2 occurred on the hominin lineage prior to the emergence of Neanderthals and Denisovans approximately 500,000 years ago. In a further study of Neanderthal FOXP2 compared to modern human FOXP2, a change in intron 8 of FOXP2, which is a binding site of POU3F2 transcription factor, was identified as differing in modern humans and Neanderthals [Maricic et al., 2013]. This change in the POU3F2 binding site could possibly alter the regulation of FOXP2 expression. In addition, genomic comparisons of Neanderthals to modern humans have identified other genomic regions as candidates for positive selection in modern humans, including genes involved in cognition, metabolism and skeletal development. In this study, 88 amino acid substitutions that have become fixed in modern humans since divergence from Neanderthals were identified [Green et al., 2010]. Thus, even small changes in human genome evolution may ultimately influence higher cognitive function and language. Such changes are particularly pertinent as many cognitive disorders are diseases unique to humans [Varki and Altheide, 2005; O'Bleness et al., 2012], indicating that they were acquired in exchange for higher cognitive function. Indeed, while genetic variation in FOXP2 itself has weak association to ASD, FOXP2 regulates genes that are disrupted in ASD, such as CNTNAP2, MET and many more [Vernes et al., 2008; Mukamel et al., 2011; Bowers and Konopka, 2012; Lepp et al., 2013]. Thus, studies of FOXP2 function should ultimately uncover some of the molecular bases underlying language and speech, notably those changes relevant to human brain evolution and cognition.

\section{FOXP2 Function and Spatiotemporal Expression}

FOXP2 is a member of the family of forkhead transcription factors, and it regulates gene expression by binding to DNA either as a homodimer or as a heterodimer with FOXP1 or FOXP4 [Li et al., 2004]. FOXP2 is 


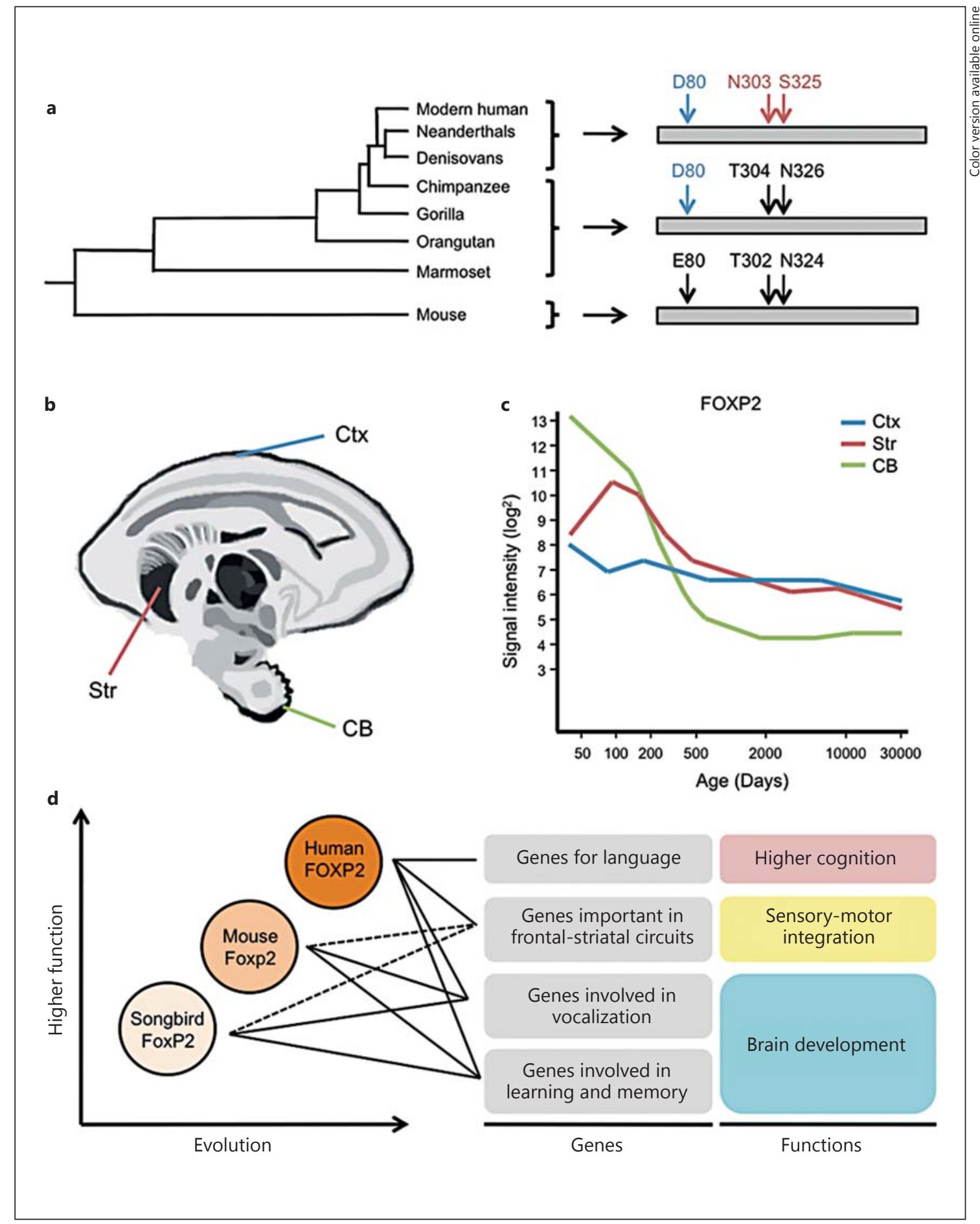

Fig. 2. Molecular evolution and function of FOXP2 in the brain. a Schematic evolutionary tree, indicating the evolution of the human genome. The diagrams show the amino acid structure of FOXP2 of modern human/Neanderthal/Denisovan, compared to chimpanzee/gorilla/orangutan/marmoset and mouse, with evolutionarily changed amino acids T303N and N325S indicated in red (right; colors refer to the online version only). A primate-specific change is also shown at D80 (blue). b FOXP2 mRNA expression is schematically represented in human fetal brain. FOXP2 expression is enriched in the striatum, deep layers of the cortex, thalamus and cerebellum. c Schematic expression of FOXP2 throughout human brain development. Data are derived from Kang et al. [2011]. d Summary diagram indicating the relationships between the molecular evolution of FOXP2 and higher cognitive functions. Ctx $=$ Cortex $;$ Str $=$ striatum; $\mathrm{CB}=$ cerebellum. 
highly expressed in the striatum and deep layers of the cortex [Ferland et al., 2003; Campbell et al., 2009] (fig. 2b, c). These regions of the human brain execute important roles in higher brain functions; for example, frontal-striatal circuits are frequently disrupted in human-specific disorders, such as ASD and schizophrenia, often through dysregulation of dopamine signaling [Hyman et al., 2006; Money and Stanwood, 2013; Russo and Nestler, 2013]. In addition, FOXP2 affects both neurogenesis, through regulation of the transition of radial glial cells to intermediate progenitors in the subventricular zone (SVZ), and neuronal differentiation, particularly neurite outgrowth during embryonic stages [Vernes et al., 2011; Tsui et al., 2013; Chiu et al., 2014]. Together, these studies suggest FOXP2 may be involved in the increase in human brain volume by regulating the number of intermediate progenitors in the inner SVZ and outer SVZ [Hansen et al., 2010]. Furthermore, FOXP2 modulates excitatory synapse density in the cortex as well as vocalization by controlling expression of the language-associated gene SRPX2, mutations of which cause disorders of language, epilepsy and cognition [Roll et al., 2006, 2010; Sia et al., 2013].

FOXP2 expression is also observed in the cerebellum, a region of the brain with a role in ASD [Becker and Stoodley, 2013]. In the cerebellum, FOXP2 expression is limited to Purkinje cells, and the dendritic arbor of these neurons is greatly diminished in Foxp2 knockout mice [Ferland et al., 2003; Shu et al., 2005; Campbell et al., 2009] (fig. 2b, c). Purkinje cells send inhibitory projections to the deep cerebellar nuclei, the sole output of all motor coordination in the cerebellum, suggesting that this critical neural pathway plays an important role in motor function and speech production. Along these lines, Foxp2 knockout mice show a reduced size of the cerebellum and disruptions to global motor function that include alteration in the ability to vocalize [Shu et al., 2005]. Taken together, FOXP2 is expressed in the right place at the right time in the brain (fig. 2c) to play a number of roles important for brain development and for orchestrating construction of brain circuits that are frequently disrupted in disorders of cognition.

\section{Functional Changes of Human FOXP2}

In order to study the functional consequences of the molecular evolution of FOXP2, Enard and colleagues generated a humanized FOXP2 mouse containing the human-specific amino acids T303N and N325S. This resulted in a number of remarkable phenotypes, including increased dendrite length and increased long-term depres- sion in medium spiny neurons of the striatum, as well as altered structure of pup isolation calls [Enard et al., 2009]. Furthermore, reduced dopamine levels were also observed in the humanized FOXP2 mouse, compared to the opposite result in Foxp2 heterozygous mice [Enard et al., 2009]. This supports the idea that human FOXP2 plays an important role in frontal-striatal circuits that have evolved in the human brain, as such circuits are often described in relation to language and speech through dopamine signaling [Ullman, 2001; Tettamanti et al., 2005; Murugan et al., 2013]. Hence, just two amino acid changes in human FOXP2 can lead to significant alterations in brain function.

Another report characterized the functional differences in transcriptional regulation by human FOXP2 compared to chimpanzee. Altered human FOXP2 function was demonstrated by conferring differential transcriptional regulation in vitro, and then these observations were extended to in vivo by using human and chimpanzee brain gene expression with network analysis to identify novel relationships among the differentially expressed genes. Notably, this study demonstrated that human FOXP2 drives differential target gene expression, and many of these targets are implicated in motor function, craniofacial formation, and cartilage and connective tissue formation, processes which may have ultimately been critical for the evolution of speech and language [Konopka et al., 2009].

As described above, FOXP2 acquired novel functions through two amino acid changes that occurred during hominin evolution. However, the role of FOXP2 is highly conserved among species for normal brain development and functions such as learning, memory and vocalization. For example, genes regulated by FOXP2 in frontal-striatal circuits for sensory-motor integration might also be similarly regulated by FoxP2 in songbird, mouse and other primates. In fact, recent work has shown that alterations in dopamine and vocalizations in songbird correlate with changes in dopamine pathwayrelated gene expression [Murugan et al., 2013], which fits with previously identified changes in dopaminerelated genes in mice and humans [Spiteri et al., 2007; Konopka et al., 2009; Vernes et al., 2011]. In contrast, we assert that the genes specifically regulated by human FOXP2 are more likely to be important for language and cognition (fig. 2d). In summary, FOXP2 is one of potentially many key genes expressed in the brain that have mediated increased cognitive function during human evolution. 


\section{Genomics Studies for Human Brain Evolution and Cognition}

\section{Approaching the Study of Human Brain Evolution}

Although FOXP2 has been shown to play a significant role in human speech and language at least partly through the acquisition of functional changes to its protein sequence, mutations in protein-coding genes cannot fully account for the cognitive differences between humans and non-human primates, as evidenced by the high similarity between the human and chimpanzee genomes revealed by sequencing [Chimpanzee Sequencing and Analysis Consortium, 2005]. It has long been postulated that changes in gene regulatory mechanisms, and therefore gene expression patterns, have driven human molecular evolution [King and Wilson, 1975], and indeed studies have shown important differences related to gene expression between humans and chimpanzees [Khaitovich et al., 2006]. Therefore, characterizing the evolution of human cognitive capabilities and disorders requires methods to analyze differences in gene regulation on the human lineage.

Examining differential expression levels of genes between humans and non-human primates can reveal important information about human brain evolution, for example that it primarily involved acceleration in the rate of gene expression changes as well as upregulation of genes [Preuss et al., 2004]. However, a limitation of differential expression analysis lies with its treatment of genes as independent entities rather than a network of players that work in concert to execute complex biological processes. Because this type of analysis results in a simple list of genes that precludes their prioritization for further study, an alternative approach is needed to tease out the relationships among genes and discover underlying structure to large-scale gene expression data sets. A recent method for this purpose is WGCNA, which takes a global approach to systematically study the interconnectedness of genes and organize them into modules with functional interpretations [Zhao et al., 2010]. By disclosing the large-scale coexpression relationships between genes, WGCNA can be a powerful tool for the study of gene regulation changes specific to human cognitive evolution and disorders.

\section{The Power of WGCNA}

In the context of evolution, the approach of WGCNA can reveal changes in gene regulation that allowed for the modification of human cognitive ability. Many gene expression relationships cannot be observed by using straightforward measures of differential gene expression, or even with added functional analyses using gene ontology approaches among primate brains. This is likely due to the fact that the relatively small evolutionary distance among primates has not permitted widely differential gene expression patterns to evolve, but small changes among groups of genes can occur and result in profound functional changes in expression in the brain. In contrast, any differential gene expression profiles that are observed among primates, especially those that change on the human lineage, are likely more highly significant in terms of functional consequences unless compensatory mechanisms have also evolved. After measuring expression levels of genes across multiple samples, for example human brain tissue, WGCNA can be applied to build a network module consisting of highly interconnected or 'hub' genes, as well as information about the direction of any given coexpressed pair of genes relative to one another (fig. 3a). Further generation of network modules from non-human primates, such as chimpanzees and macaques, can reveal gene relationships that arose on the human lineage after its split from the other lineages (fig. 3a). In addition to showing the connectedness of genes within a module, WGCNA can lead to comparisons between modules and expose evolutionary relationships among primates. For example, between humans and chimpanzees, analysis of frontal pole (FP) and caudate nucleus $(\mathrm{CN})$ module preservation reveals less conservation of FP modules than $\mathrm{CN}$ modules, reflecting the recent expansion and modification of FP in human evolution [Dumontheil et al., 2008; Semendeferi et al., 2011; Konopka et al., 2012b] (fig. 3b). This pattern of FP and $\mathrm{CN}$ module preservation also arises after comparison of humans and macaques, and this comparison shows an overall lower module preservation, reflecting the larger evolutionary distance between humans and macaques [Konopka et al., 2012b] (fig. 3b).

Thus far, WGCNA has been employed in several studies comparing humans and non-human primates in order to gain insights into human brain evolution. A comparison of human and chimpanzee brain regions resulted in the identification of human-specific network connections, and also showed that module conservation in the cortex is weaker relative to subcortical areas in accordance with known evolutionary hierarchies [Oldham et al., 2006]. A later study utilized the power of NGS to find that, relative to both chimpanzee and macaque, the human frontal lobe exhibits differential expression of genes as well as an increase in transcriptional complexity [Konopka et al., 2012b]. Moreover, a human-specific frontal 
lobe module containing the circadian gene CLOCK was identified, and another frontal lobe module containing genes coexpressed with FOXP2 was found to be enriched for neuronal morphological processes [Konopka et al., 2012b]. Human-specific patterns of gene expression during brain development have been identified by a recent analysis of the fetal human neocortex, and hub genes from these developmental modules have been examined in fetal macaque brain tissue to identify potentially conserved or evolved functional relevance [Pletikos et al., 2014]. In addition to identifying gene expression differences between species, WGCNA has been used to uncover modules within the human brain relating to cell type [Oldham et al., 2008] and fetal development [Johnson et al., 2009] that can be queried for future studies across primates. Altogether, these studies demonstrate the utility of WGCNA in revealing patterns of gene expression not evident based on differential expression analysis alone, and they provide evidence that gene networks between and within brain regions undergo remodeling over the course of evolution.

WGCNA has also been utilized to investigate the genetic bases for human-specific cognitive disorders that are difficult to model using traditional approaches. One study demonstrating the utility of human neuronal progenitors revealed that a significant number of ASD candidate genes are coregulated over the course of neuronal differentiation, providing insight into the signaling pathways disrupted in ASD [Konopka et al., 2012a]. Multiple studies using postmortem brain from patients with ASD or schizophrenia have found differences in gene coexpression networks relating to neurodevelopmental processes [Torkamani et al., 2010; Voineagu et al., 2011; Roussos et al., 2012; Chen et al., 2013]. In addition, WGCNA has been applied to study neurodegenerative disorders including Alzheimer's disease [Miller et al., 2008, 2010] and frontotemporal dementia [Rosen et al., 2011]. More recent studies have employed both WGCNA as well as other similar network methods to synthesize genetic information with spatial and temporal gene expression transcriptomes in the human brain [Parikshak et al., 2013; Willsey et al., 2013; Liu et al., 2014; Tebbenkamp et al., 2014]. These studies make critical insights into disease biology by uncovering patterns of gene expression linked with specific genetic etiologies in ASD and other neurodevelopmental disorders. Identification of the gene networks disrupted in human-specific disorders will provide targets for therapeutic treatment as well as insights into the evolution of human-specific cognitive functions.

Decoding the Molecular Evolution of Human Cognition

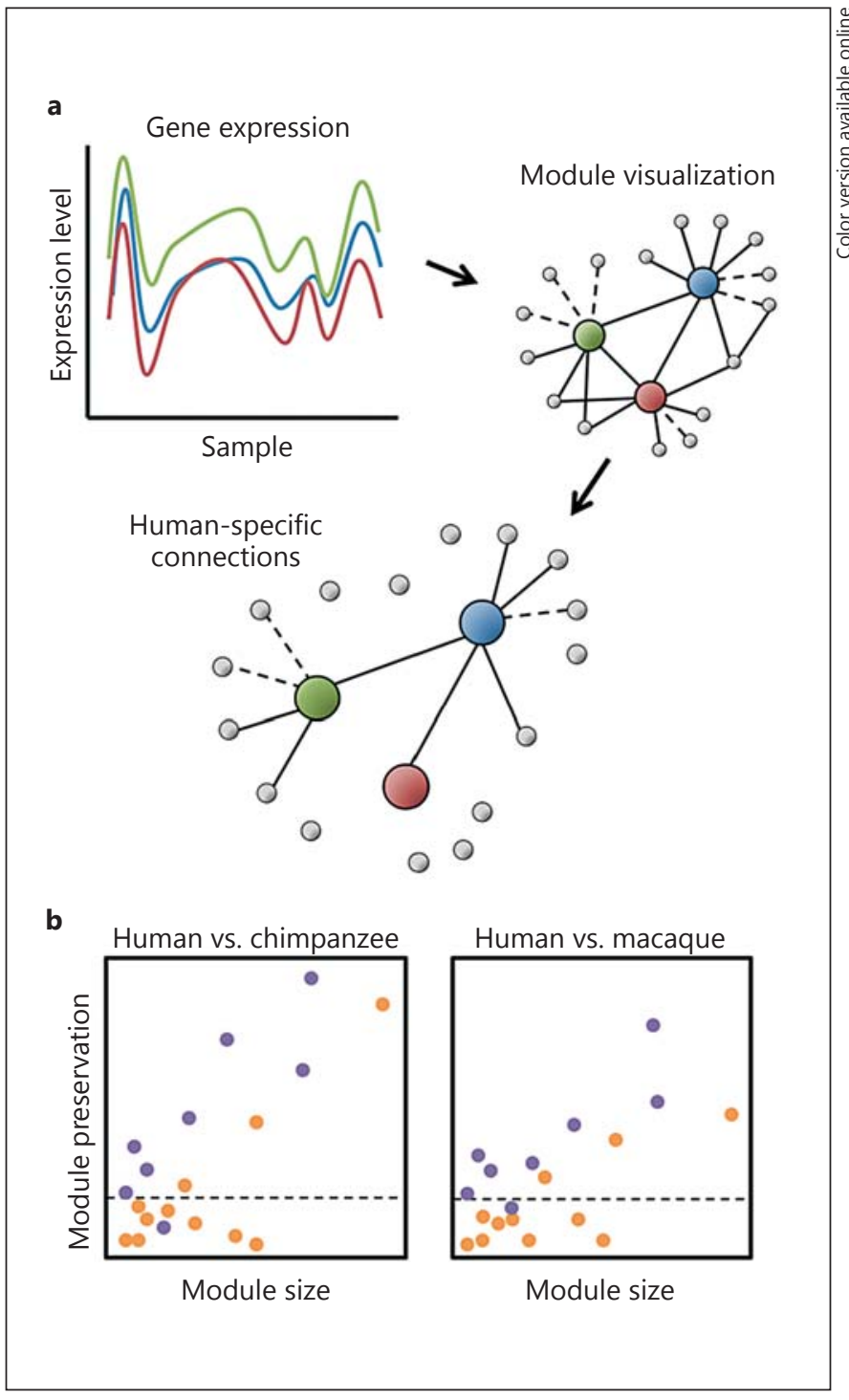

Fig. 3. Use of WGCNA to identify human-specific features. a In this schematic, coexpression levels of human brain genes across samples are measured to build a network module. Hub genes are depicted as green, blue and red nodes, and additional genes in the network are depicted as grey nodes (colors refer to the online version only). Solid lines indicate positive correlations, and dashed lines indicate negative correlations. Comparisons of network modules generated from human brain and chimpanzee brain can reveal human-specific connections between genes. b Module preservation comparisons expose evolutionary relationships among primates. In this example dataset, roughly modeled after Konopka et al. [2012b], modules are more highly conserved between human and chimpanzee than between human and macaque, and FP modules (orange) tend to be less conserved than $\mathrm{CN}$ modules (purple). Modules below the dashed line are not preserved and are human specific. 


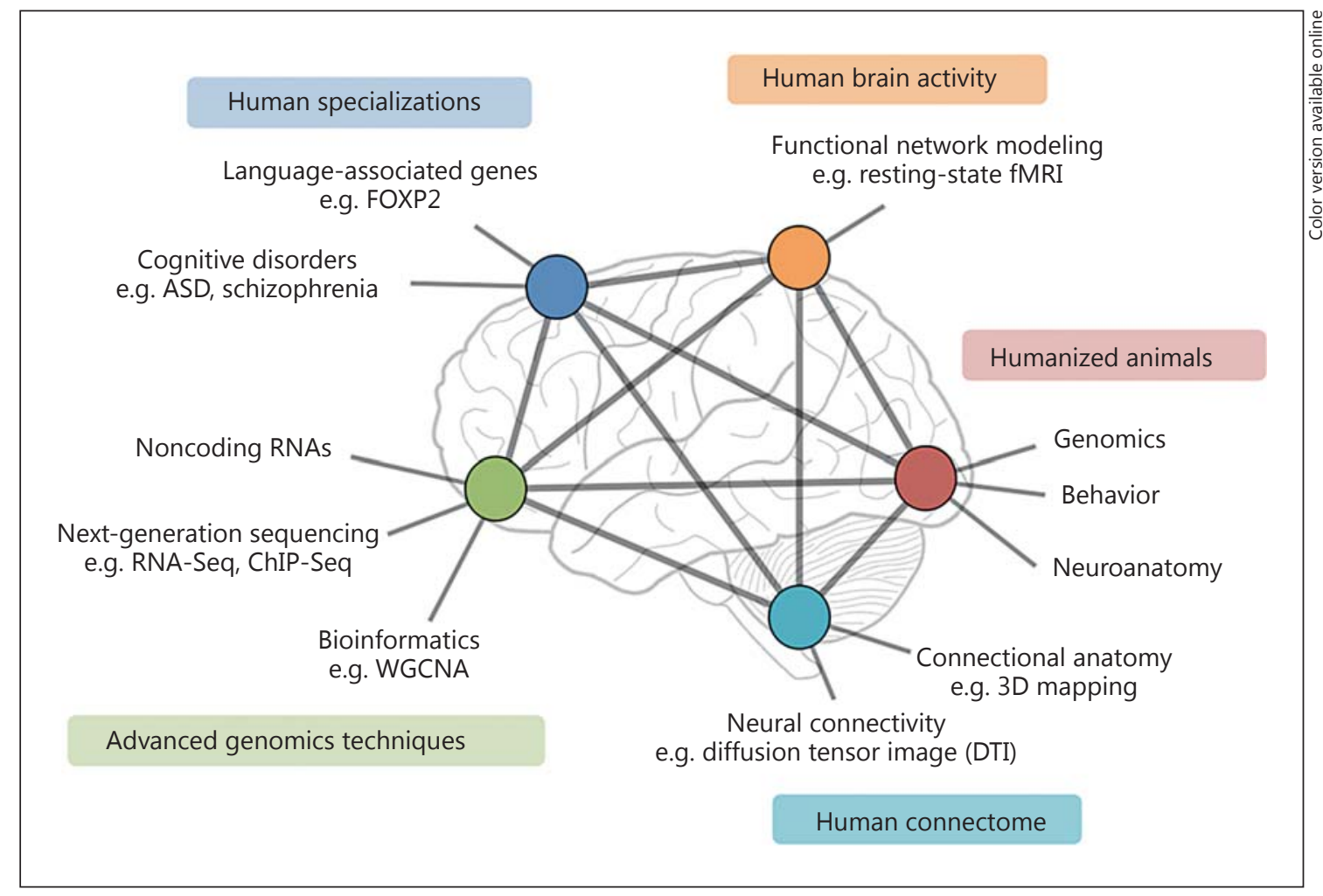

Fig. 4. Future directions for understanding human brain evolution. Summary diagram showing future directions of evolutionary approaches to human brain evolution, cognition and disease. In addition to current evolutionary approaches, such as human specializations, advanced genomic techniques and humanized ani-

\section{Future Directions for Studies at the Functional} Genomics Level

A wealth of information regarding the origins of human cognition remains to be uncovered through the use of advanced genomics techniques. Previous identification of human-specific gene expression patterns prompts further investigation of their effects on brain function. As mentioned before, the central gene in a human-specific FP network is CLOCK, a circadian gene encoding a transcription factor that is implicated in neuropsychiatric disorders [Coque et al., 2011; Menet and Rosbash, 2011; Konopka et al., 2012b]. Thus, identifying CLOCK transcriptional targets and patterns of regulation unique to the human brain should prove informative for understanding human evolution and disorders. Additionally, the human genome has demonstrated accelerated recruitment of newly emergent brain development genes [Zhang et al., 2011], prompting the question of whether these young genes contribute more to human-specific neocortex evolution or to overall primate neocortex evolution. mals, human brain activity information based on resting-state fMRI studies and Human Connectome Project data will be combined to provide a greater understanding of human cognitive function.

Another approach to studying human evolution may be to identify physiological features uniquely altered on the human lineage and to design strategies that elucidate the genetic programs mediating their development and function. For example, dendritic spines are increased both in number [Elston et al., 2001] and density [Duan et al., 2003] on human neurons compared to macaque neurons. In addition, humans have increased numbers of spines and dendritic branching compared to chimpanzees, although spine density is not different [Bianchi et al., 2013], and human-specific duplications of SRGAP2 have been linked to changes in spine maturation [Charrier et al., 2012; Dennis et al., 2012]. Network analysis may provide additional molecular correlates for these anatomical differences, which can be functionally investigated through the use of primary cultures, such as human neural progenitors. In addition, recent advances using induced pluripotent stem cells from a range of primates, including human, chimpanzee, gorilla and bonobo, have demonstrated the utility of 
these cells for the study of molecular evolution [Marchetto et al., 2013; Wunderlich et al., 2014]. Induced pluripotent stem cells from multiple primate species that are induced to become neurons can be harnessed for studying the role of evolutionarily relevant genes on neuronal differentiation and function.

\section{Future Directions}

Comparative genomics studies of the primate brain have been limited by the availability of appropriate highquality tissue samples from humans and great apes. Unfortunately, this problem will likely only continue to worsen as chimpanzee breeding is no longer supported by any government agency and other primate species face imminent extinction. With remaining tissues, however, it will be important to expand the regions queried as well as the developmental time points examined. In addition, gene expression profiling at the single cell level should resolve the cellular heterogeneity issue at play in most of the brain. Finally, although not discussed in detail here, increased knowledge of the comparative regulatory regions of the genome, including the epigenome, and noncoding RNAs will certainly add to our under- standing of human-relevant gene expression and regulation. Furthermore, novel insights into mapping connectivity and activity in the human brain by the Human Connectome Project together with resting-state fMRI or diffusion tensor image (DTI) studies will be informative as well (fig. 4). Diverse approaches that marry expression, whether it be by using RNA-seq, in situ hybridization or immunohistochemistry, together with functional and connectivity imaging studies, will ultimately lead to a treasure trove of knowledge about human brain evolution and cognition. Translating this information into working knowledge of the brain that can be harnessed for therapeutic development will be challenging, but not unachievable. By utilizing information about our brains' evolutionary history, we will hopefully change our futures through the treatment and potential cure of cognitive disorders.

\section{Acknowledgements}

N.U. is a research fellow of The Uehara Memorial Foundation. G.K. is a Jon Heighten Scholar in Autism Research at UT Southwestern. This work was supported by the NIMH (R00MH090238), a March of Dimes Basil O’Connor Starter Scholar Research Award, Once Upon a Time Foundation, and CREW Dallas to G.K.

\section{References}

Allman J, Tetreault NA, Hakeem AY, Manaye KF, Semendeferi K, Erwin JM, Park S, Goubert V, Hof PR (2010): The von Economo neurons in frontoinsular and anterior cingulate cortex in great apes and humans. Brain Struct Funct 214:495-517.

American Psychiatric Association (2000): Diagnostic and Statistical Manual of Mental Disorders, ed 4, text revision (DSM-IV-TR). Arlington, APA.

American Psychiatric Association (2013): Diagnostic and Statistical Manual of Mental Disorders, ed 5 (DSM-5). Arlington, APA.

Bacon C, Rappold GA (2012): The distinct and overlapping phenotypic spectra of FOXP1 and FOXP2 in cognitive disorders. Hum Genet 131:1687-1698.

Baird G, Simonoff E, Pickles A, Chandler S, Loucas T, Meldrum D, Charman T (2006): Prevalence of disorders of the autism spectrum in a population cohort of children in South Thames: the Special Needs and Autism Project (SNAP). Lancet 368:210-215.

- Ballesteros-Yáñez I, Benavides-Piccione R, Elston GN, Yuste R, DeFelipe J (2006): Density and morphology of dendritic spines in mouse neocortex. Neuroscience 138:403-409.
Becker EB, Stoodley CJ (2013): Autism spectrum disorder and the cerebellum. Int Rev Neurobiol 113:1-34.

-Berg JM, Geschwind DH (2012): Autism genetics: searching for specificity and convergence. Genome Biol 13:247.

Bianchi S, Stimpson CD, Bauernfeind AL, Schapiro SJ, Baze WB, McArthur MJ, Bronson E, Hopkins WD, Semendeferi K, Jacobs B, Hof PR, Sherwood CC (2013): Dendritic morphology of pyramidal neurons in the chimpanzee neocortex: regional specializations and comparison to humans. Cereb Cortex 23 : 2429-2436.

Bowers JM, Konopka G (2012): The role of the FOXP family of transcription factors in ASD. Dis Markers 33:251-260.

Bruner E, Jacobs HI (2013): Alzheimer's disease: the downside of a highly evolved parietal lobe? J Alzheimers Dis 35:227-240.

Buckner RL, Krienen FM (2013): The evolution of distributed association networks in the human brain. Trends Cogn Sci 17:648-665.
Campbell P, Reep RL, Stoll ML, Ophir AG, Phelps SM (2009): Conservation and diversity of Foxp2 expression in muroid rodents: functional implications. J Comp Neurol 512:84100.

Chakrabarti S, Fombonne E (2001): Pervasive developmental disorders in preschool children. JAMA 285:3093-3099.

Charrier C, Joshi K, Coutinho-Budd J, Kim JE, Lambert N, de Marchena J, Jin WL, Vanderhaeghen P, Ghosh A, Sassa T, Polleux F (2012): Inhibition of SRGAP2 function by its human-specific paralogs induces neoteny during spine maturation. Cell 149:923-935.

-Chen C, Cheng L, Grennan K, Pibiri F, Zhang C, Badner JA, Gershon ES, Liu C (2013): Two gene co-expression modules differentiate psychotics and controls. Mol Psychiatry 18: 1308-1314.

-Chimpanzee Sequencing and Analysis Consortium (2005): Initial sequence of the chimpanzee genome and comparison with the human genome. Nature 437:69-87.

Chiu YC, Li MY, Liu YH, Ding JY, Yu JY, Wang TW (2014): Foxp2 regulates neuronal differentiation and neuronal subtype specification. Dev Neurobiol 74:723-728.
Decoding the Molecular Evolution of Human Cognition 
Coque L, Mukherjee S, Cao JL, Spencer S, Marvin M, Falcon E, Sidor MM, Birnbaum SG, Graham A, Neve RL, Gordon E, Ozburn AR, Goldberg MS, Han MH, Cooper DC, McClung CA (2011): Specific role of VTA dopamine neuronal firing rates and morphology in the reversal of anxiety-related, but not depression-related behavior in the Clock $\Delta 19$ mouse model of mania. Neuropsychopharmacology 36:1478-1488.

Crawley JN (2012): Translational animal models of autism and neurodevelopmental disorders. Dialogues Clin Neurosci 14:293-305.

-Crespi B, Summers K, Dorus S (2007): Adaptive evolution of genes underlying schizophrenia. Proc Biol Sci 274:2801-2810.

Crow TJ (2000): Schizophrenia as the price that homo sapiens pays for language: a resolution of the central paradox in the origin of the species. Brain Res Brain Res Rev 31:118-129.

Crow TJ (2008): The 'big bang' theory of the origin of psychosis and the faculty of language. Schizophr Res 102:31-52.

Dennis MY, Nuttle X, Sudmant PH, Antonacci F, Graves TA, Nefedov M, Rosenfeld JA, Sajjadian S, Malig M, Kotkiewicz H, Curry CJ, Shafer S, Shaffer LG, de Jong PJ, Wilson RK, Eichler EE (2012): Evolution of human-specific neural SRGAP2 genes by incomplete segmental duplication. Cell 149:912-922.

-Doody RS, Thomas RG, Farlow M, et al (2014): Phase 3 trials of solanezumab for mild-tomoderate Alzheimer's disease. N Engl J Med 370:311-321.

Duan H, Wearne SL, Rocher AB, Macedo A, Morrison JH, Hof PR (2003): Age-related dendritic and spine changes in corticocortically projecting neurons in macaque monkeys. Cereb Cortex 13:950-961.

Dumontheil I, Burgess PW, Blakemore SJ (2008): Development of rostral prefrontal cortex and cognitive and behavioural disorders. Dev Med Child Neurol 50:168-181.

-Elsabbagh M, Divan G, Koh YJ, Kim YS, Kauchali S, Marcín C, Montiel-Nava C, Patel V, Paula CS, Wang C, Yasamy MT, Fombonne E (2012): Global prevalence of autism and other pervasive developmental disorders. Autism Res 5:160-179.

-Elston GN, Benavides-Piccione R, DeFelipe J (2001): The pyramidal cell in cognition: a comparative study in human and monkey. J Neurosci 21:RC163.

-Enard W, Gehre S, Hammerschmidt K, et al (2009): A humanized version of Foxp2 affects cortico-basal ganglia circuits in mice. Cell 137:961-971.

-Enard W, Przeworski M, Fisher SE, Lai CS, Wiebe V, Kitano T, Monaco AP, Pääbo S (2002): Molecular evolution of FOXP2, a gene involved in speech and language. Nature 418:869-872.

- Ferland RJ, Cherry TJ, Preware PO, Morrisey EE, Walsh CA (2003): Characterization of Foxp2 and Foxp1 mRNA and protein in the developing and mature brain. J Comp Neurol 460: 266-279.
Fernell E, Gillberg C (2010): Autism spectrum disorder diagnoses in Stockholm preschoolers. Res Dev Disabil 31:680-685.

Fisher SE, Lai CS, Monaco AP (2003): Deciphering the genetic basis of speech and language disorders. Annu Rev Neurosci 26:57-80.

Flavell SW, Cowan CW, Kim TK, Greer PL, Lin Y, Paradis S, Griffith EC, Hu LS, Chen C, Greenberg ME (2006): Activity-dependent regulation of MEF2 transcription factors suppresses excitatory synapse number. Science 311:1008-1012.

Fombonne E (2005): Epidemiology of autistic disorder and other pervasive developmental disorders. J Clin Psychiatry 66(suppl 10):3-8.

Green RE, Krause J, Briggs AW, et al (2010): A draft sequence of the Neandertal genome. Science 328:710-722.

- Hansen DV, Lui JH, Parker PR, Kriegstein AR (2010): Neurogenic radial glia in the outer subventricular zone of human neocortex. $\mathrm{Na}-$ ture 464:554-561.

-Hyman SE, Malenka RC, Nestler EJ (2006): Neural mechanisms of addiction: the role of reward-related learning and memory. Annu Rev Neurosci 29:565-598.

Johnson MB, Kawasawa YI, Mason CE, Krsnik Z, Coppola G, Bogdanovic D, Geschwind DH, Mane SM, State MW, Sestan N (2009): Functional and evolutionary insights into human brain development through global transcriptome analysis. Neuron 62:494-509.

Kang HJ, Kawasawa YI, Cheng F, Zhu Y, Xu X, Li M, Sousa AM, Pletikos M, Meyer KA, Sedmak G, Guennel T, Shin Y, Johnson MB, Krsnik Z, Mayer S, Fertuzinhos S, Umlauf S, Lisgo SN, Vortmeyer A, Weinberger DR, Mane S, Hyde TM, Huttner A, Reimers M, Kleinman JE, Sestan N (2011): Spatio-temporal transcriptome of the human brain. Nature 478:483489.

Kelleher RJ 3rd, Bear MF (2008): The autistic neuron: troubled translation? Cell 135:401-406.

Khaitovich P, Enard W, Lachmann M, Pääbo S (2006): Evolution of primate gene expression. Nat Rev Genet 7:693-702.

Kim YS, State MW (2014): Recent challenges to the psychiatric diagnostic nosology: a focus on the genetics and genomics of neurodevelopmental disorders. Int J Epidemiol 43:465475.

King BH, Lord C (2011): Is schizophrenia on the autism spectrum? Brain Res 1380:34-41.

King MC, Wilson AC (1975): Evolution at two levels in humans and chimpanzees. Science 188:107-116.

Konopka G, Bomar JM, Winden K, Coppola G, Jonsson ZO, Gao F, Peng S, Preuss TM, Wohlschlegel JA, Geschwind DH (2009): Human-specific transcriptional regulation of CNS development genes by FOXP2. Nature 462:213-217.

Konopka G, Friedrich T, Davis-Turak J, Winden K, Oldham MC, Gao F, Chen L, Wang GZ, Luo R, Preuss TM, Geschwind DH (2012b): Human-specific transcriptional networks in the brain. Neuron 75:601-617.
Konopka G, Geschwind DH (2010): Human brain evolution: harnessing the genomics (r) evolution to link genes, cognition, and behavior. Neuron 68:231-244.

Konopka G, Wexler E, Rosen E, Mukamel Z, Osborn GE, Chen L, Lu D, Gao F, Gao K, Lowe JK, Geschwind DH (2012a): Modeling the functional genomics of autism using human neurons. Mol Psychiatry 17:202-214.

Krause J, Lalueza-Fox C, Orlando L, Enard W, Green RE, Burbano HA, Hublin JJ, Hänni C, Fortea J, de la Rasilla M, Bertranpetit J, Rosas A, Pääbo S (2007): The derived FOXP2 variant of modern humans was shared with Neandertals. Curr Biol 17:1908-1912.

Krumm N, O’Roak BJ, Shendure J, Eichler EE (2014): A de novo convergence of autism genetics and molecular neuroscience. Trends Neurosci 37:95-105.

Lai CS, Fisher SE, Hurst JA, Vargha-Khadem F, Monaco AP (2001): A forkhead-domain gene is mutated in a severe speech and language disorder. Nature 413:519-523.

Lepp S, Anderson A, Konopka G (2013): Connecting signaling pathways underlying communication to ASD vulnerability. Int Rev Neurobiol 113:97-133.

Li G, Wang J, Rossiter SJ, Jones G, Zhang S (2007): Accelerated FoxP2 evolution in echolocating bats. PLoS One 2:e900.

Li Q, Bian S, Liu B, Hong J, Toth M, Sun T (2013): Establishing brain functional laterality in adult mice through unilateral gene manipulation in the embryonic cortex. Cell Res 23: 1147-1149.

Li S, Weidenfeld J, Morrisey EE (2004): Transcriptional and DNA binding activity of the Foxp1/2/4 family is modulated by heterotypic and homotypic protein interactions. Mol Cell Biol 24:809-822.

Liu L, Lei J, Sanders SJ, Willsey AJ, Kou Y, Cicek AE, Klei L, Lu C, He X, Li M, Muhle RA, Ma'ayan A, Noonan JP, Sestan N, McFadden KA, State MW, Buxbaum JD, Devlin B, Roeder K (2014): DAWN: a framework to identify autism genes and subnetworks using gene expression and genetics. Mol Autism 5:22.

Marchetto MC, Narvaiza I, Denli AM, Benner C, Lazzarini TA, Nathanson JL, Paquola AC, Desai KN, Herai RH, Weitzman MD, Yeo GW, Muotri AR, Gage FH (2013): Differential $\mathrm{L} 1$ regulation in pluripotent stem cells of humans and apes. Nature 503:525-529.

-Marcus GF, Fisher SE (2003): FOXP2 in focus: what can genes tell us about speech and language? Trends Cogn Sci 7:257-262.

Maricic T, Günther V, Georgiev O, Gehre S, Curlin $\mathrm{M}$, Schreiweis C, Naumann R, Burbano HA, Meyer M, Lalueza-Fox C, de la Rasilla M, Rosas A, Gajovic S, Kelso J, Enard W, Schaffner W, Pääbo S (2013): A recent evolutionary change affects a regulatory element in the human FOXP2 gene. Mol Biol Evol 30:844-852. 
Menet JS, Rosbash M (2011): When brain clocks lose track of time: cause or consequence of neuropsychiatric disorders. Curr Opin Neurobiol 21:849-857.

Miller JA, Horvath S, Geschwind DH (2010): Divergence of human and mouse brain transcriptome highlights Alzheimer disease pathways. Proc Natl Acad Sci USA 107:1269812703.

-Miller JA, Oldham MC, Geschwind DH (2008): A systems level analysis of transcriptional changes in Alzheimer's disease and normal aging. J Neurosci 28:1410-1420.

-Money KM, Stanwood GD (2013): Developmental origins of brain disorders: roles for dopamine. Front Cell Neurosci 7:260.

-Mukamel Z, Konopka G, Wexler E, Osborn GE, Dong H, Bergman MY, Levitt P, Geschwind DH (2011): Regulation of MET by FOXP2, genes implicated in higher cognitive dysfunction and autism risk. J Neurosci 31:1143711442 .

-Murugan M, Harward S, Scharff C, Mooney R (2013): Diminished FoxP2 levels affect dopaminergic modulation of corticostriatal signaling important to song variability. Neuron 80 : 1464-1476.

O'Bleness M, Searles VB, Varki A, Gagneux P, Sikela JM (2012): Evolution of genetic and genomic features unique to the human lineage. Nat Rev Genet 13:853-866.

-Oh SW, Harris JA, Ng L, et al (2014): A mesoscale connectome of the mouse brain. Nature 508: 207-214.

- Oldham MC, Horvath S, Geschwind DH (2006): Conservation and evolution of gene coexpression networks in human and chimpanzee brains. Proc Natl Acad Sci USA 103:1797317978.

-Oldham MC, Konopka G, Iwamoto K, Langfelder P, Kato T, Horvath S, Geschwind DH (2008): Functional organization of the transcriptome in human brain. Nat Neurosci 11:1271-1282.

- Parikshak NN, Luo R, Zhang A, Won H, Lowe JK, Chandran V, Horvath S, Geschwind DH (2013): Integrative functional genomic analyses implicate specific molecular pathways and circuits in autism. Cell 155:1008-1021.

-Pletikos M, Sousa AM, Sedmak G, Meyer KA, Zhu Y, Cheng F, Li M, Kawasawa YI, Sestan N (2014): Temporal specification and bilaterality of human neocortical topographic gene expression. Neuron 81:321-332.

-Preuss TM, Cáceres M, Oldham MC, Geschwind DH (2004): Human brain evolution: insights from microarrays. Nat Rev Genet 5:850-860.

-Raichlen DA, Alexander GE (2014): Exercise, APOE genotype, and the evolution of the human lifespan. Trends Neurosci 37:247-255.
Reich D, Green RE, Kircher M, Krause J, Patterson N, Durand EY, Viola B, Briggs AW, Stenzel U, Johnson PL, Maricic T, Good JM, Marques-Bonet T, Alkan C, Fu Q, Mallick S, Li H, Meyer M, Eichler EE, Stoneking M, Richards M, Talamo S, Shunkov MV, Derevianko AP, Hublin JJ, Kelso J, Slatkin M, Pääbo S (2010): Genetic history of an archaic hominin group from Denisova Cave in Siberia. Nature 468:1053-1060.

Robertson MM (2008): The prevalence and epidemiology of Gilles de la Tourette syndrome. Part 1: the epidemiological and prevalence studies. J Psychosom Res 65:461-472.

Roll P, Rudolf G, Pereira S, et al (2006): SRPX2 mutations in disorders of language cortex and cognition. Hum Mol Genet 15:1195-1207.

Roll P, Vernes SC, Bruneau N, Cillario J, PonsoleLenfant M, Massacrier A, Rudolf G, Khalife M, Hirsch E, Fisher SE, Szepetowski P (2010): Molecular networks implicated in speech-related disorders: FOXP2 regulates the SRPX2/ uPAR complex. Hum Mol Genet 19:48484860.

-Rosen EY, Wexler EM, Versano R, Coppola G, Gao F, Winden KD, Oldham MC, Martens LH, Zhou P, Farese RV Jr, Geschwind DH (2011): Functional genomic analyses identify pathways dysregulated by progranulin deficiency, implicating Wnt signaling. Neuron 71:1030-1042.

Roussos P, Katsel P, Davis KL, Siever LJ, Haroutunian V (2012): A system-level transcriptomic analysis of schizophrenia using postmortem brain tissue samples. Arch Gen Psychiatry 69:1205-1213.

Russo SJ, Nestler EJ (2013): The brain reward circuitry in mood disorders. Nat Rev Neurosci 14:609-625.

Salloway S, Sperling R, Fox NC, et al (2014): Two phase 3 trials of bapineuzumab in mild-tomoderate Alzheimer's disease. N Engl J Med 370:322-333.

Sanders SJ, Murtha MT, Gupta AR, Murdoch JD, Raubeson MJ, Willsey AJ, Ercan-Sencicek AG, DiLullo NM, Parikshak NN, Stein JL, Walker MF, Ober GT, Teran NA, Song Y, ElFishawy P, Murtha RC, Choi M, Overton JD, Bjornson RD, Carriero NJ, Meyer KA, Bilguvar K, Mane SM, Sestan N, Lifton RP, Günel M, Roeder K, Geschwind DH, Devlin B, State MW (2012): De novo mutations revealed by whole-exome sequencing are strongly associated with autism. Nature 485:237-241.

-Semendeferi K, Teffer K, Buxhoeveden DP, Park MS, Bludau S, Amunts K, Travis K, Buckwalter J (2011): Spatial organization of neurons in the frontal pole sets humans apart from great apes. Cereb Cortex 21:1485-1497.

-Seyfarth RM, Cheney DL (2013): Affiliation, empathy, and the origins of theory of mind. Proc Natl Acad Sci USA 110(suppl 2):1034910356.

-Shettleworth SJ (2012): Modularity, comparative cognition and human uniqueness. Philos Trans R Soc Lond B Biol Sci 367:2794-2802.
Shu W, Cho JY, Jiang Y, Zhang M, Weisz D, Elder GA, Schmeidler J, De Gasperi R, Sosa MA, Rabidou D, Santucci AC, Perl D, Morrisey E, Buxbaum JD (2005): Altered ultrasonic vocalization in mice with a disruption in the Foxp2 gene. PNAS 102:9643-9648.

Sia GM, Clem RL, Huganir RL (2013): The human language-associated gene SRPX2 regulates synapse formation and vocalization in mice. Science 342:987-991.

- Silverman JL, Yang M, Lord C, Crawley JN (2010): Behavioural phenotyping assays for mouse models of autism. Nat Rev Neurosci 11:490502.

- Somel M, Liu X, Khaitovich P (2013): Human brain evolution: transcripts, metabolites and their regulators. Nat Rev Neurosci 14:112-127.

- Spiteri E, Konopka G, Coppola G, Bomar J, Oldham M, Ou J, Vernes SC, Fisher SE, Ren B, Geschwind DH (2007): Identification of the transcriptional targets of FOXP2, a gene linked to speech and language, in developing human brain. Am J Hum Genet 81:11441157.

Tebbenkamp AT, Willsey AJ, State MW, Sestan N (2014): The developmental transcriptome of the human brain: implications for neurodevelopmental disorders. Curr Opin Neurol 27: 149-156.

Teffer K, Semendeferi K (2012): Human prefrontal cortex: evolution, development, and pathology. Prog Brain Res 195:191-218.

Telese F, Gamliel A, Skowronska-Krawczyk D, Garcia-Bassets I, Rosenfeld MG (2013): 'Seq-ing' insights into the epigenetics of neuronal gene regulation. Neuron 77:606-623.

Tettamanti M, Moro A, Messa C, Moresco RM, Rizzo G, Carpinelli A, Matarrese M, Fazio F, Perani D (2005): Basal ganglia and language: phonology modulates dopaminergic release. Neuroreport 16:397-401.

- Torkamani A, Dean B, Schork NJ, Thomas EA (2010): Coexpression network analysis of neural tissue reveals perturbations in developmental processes in schizophrenia. Genome Res 20:403-412.

Toro R, Konyukh M, Delorme R, Leblond C, Chaste P, Fauchereau F, Coleman M, Leboyer M, Gillberg C, Bourgeron T (2010): Key role for gene dosage and synaptic homeostasis in autism spectrum disorders. Trends Genet 26: 363-372.

Tsui D, Vessey JP, Tomita H, Kaplan DR, Miller FD (2013): FoxP2 regulates neurogenesis during embryonic cortical development. J Neurosci 33:244-258.

Ullman MT (2001): A neurocognitive perspective on language: the declarative/procedural model. Nat Rev Neurosci 2:717-726.

Vargha-Khadem F, Gadian DG, Copp A, Mishkin M (2005): FOXP2 and the neuroanatomy of speech and language. Nat Rev Neurosci 6: 131-138.
Decoding the Molecular Evolution of Human Cognition
Brain Behav Evol 2014;84:103-116 DOI: $10.1159 / 000365182$ 
Varki A, Altheide TK (2005): Comparing the human and chimpanzee genomes: searching for needles in a haystack. Genome Res 15:17461758.

-Vernes SC, Newbury DF, Abrahams BS, Winchester L, Nicod J, Groszer M, Alarcón M, Oliver PL, Davies KE, Geschwind DH, Monaco AP, Fisher SE (2008): A functional genetic link between distinct developmental language disorders. N Engl J Med 359:23372345.

-Vernes SC, Oliver PL, Spiteri E, Lockstone HE, Puliyadi R, Taylor JM, Ho J, Mombereau C, Brewer A, Lowy E, Nicod J, Groszer M, Baban D, Sahgal N, Cazier JB, Ragoussis J, Davies KE, Geschwind DH, Fisher SE (2011): Foxp2 regulates gene networks implicated in neurite outgrowth in the developing brain. PLoS Genet 7:e1002145.
Voineagu I, Wang X, Johnston P, Lowe JK, Tian Y, Horvath S, Mill J, Cantor RM, Blencowe BJ, Geschwind DH (2011): Transcriptomic analysis of autistic brain reveals convergent molecular pathology. Nature 474:380-384.

Werling DM, Geschwind DH (2013): Sex differences in autism spectrum disorders. Curr Opin Neurol 26:146-153.

Willsey AJ, Sanders SJ, Li M, Dong S, Tebbenkamp AT, Muhle RA, Reilly SK, Lin L, Fertuzinhos S, Miller JA, Murtha MT, Bichsel C, Niu W, Cotney J, Ercan-Sencicek AG, Gockley J, Gupta AR, Han W, He X, Hoffman EJ, Klei L, Lei J, Liu W, Liu L, Lu C, Xu X, Zhu Y, Mane SM, Lein ES, Wei L, Noonan JP, Roeder K, Devlin B, Sestan N, State MW (2013): Coexpression networks implicate human midfetal deep cortical projection neurons in the pathogenesis of autism. Cell 155:997-1007.
-Wunderlich S, Kircher M, Vieth B, Haase A, Merkert S, Beier J, Göhring G, Glage S, Schambach A, Curnow EC, Pääbo S, Martin U, Enard W (2014): Primate iPS cells as tools for evolutionary analyses. Stem Cell Res 12: 622-629.

Zhang YE, Landback P, Vibranovski MD, Long M (2011): Accelerated recruitment of new brain development genes into the human genome. PLoS Biol 9:e1001179.

Zhao W, Langfelder P, Fuller T, Dong J, Li A, Hovarth S (2010): Weighted gene coexpression network analysis: state of the art. J Biopharm Stat 20:281-300. 